\title{
Exclusive breastfeeding and its impact on neurodevelopment
}

\author{
Saeed Salem Alghamdi ${ }^{1 *}$, Mansour Sultan Alruwaili², Nafea Hamoud Alanazi², \\ Abdulaziz Zaher Alalmaei ${ }^{2}$, Rashed Sattam Alshammari ${ }^{2}$, Faisal Fahad Bin Tuwalah ${ }^{2}$, \\ Abdullah Saif Alqahtani², Humoud Auoad Alhoraim², Omar Mohammad Alshammari², \\ Munirah Mansour Alamro², Abdulrahman Saleh Almisfer ${ }^{2}$
}

\author{
${ }^{1}$ Department of Pediatrics, Al Aziziyah Children Hospital, Jeddah, Saudi Arabia \\ ${ }^{2}$ Department of Pediatrics, Al Yamamah Hospital, Riyadh, Saudi Arabia
}

Received: 08 July 2021

Accepted: 23 July 2021

\author{
*Correspondence: \\ Dr. Saeed Salem Alghamdi, \\ E-mail: Saeed_001@hotmail.com
}

Copyright: () the author(s), publisher and licensee Medip Academy. This is an open-access article distributed under the terms of the Creative Commons Attribution Non-Commercial License, which permits unrestricted non-commercial use, distribution, and reproduction in any medium, provided the original work is properly cited.

\begin{abstract}
Many advantages have been reported among the various studies in the literature about the efficacy of breastfeeding on brain development, which is mainly attributable to the abundant presence of the bioactive compounds and the essential fatty acids in breast milk. Moreover, it has been proven that breastfeeding enhanced long-term outcomes in children. In this context, arachidonic acid and docosahexaenoic acid are two fatty acids that were previously reported to be associated with the developmental outcomes of the retina, nerve cells and brain. Both of these amino acids have also been found in breast milk, and they are not present in other milk formulas as cow's milk which is usually administered to newborns. In the present literature review, we aim to discuss the impact of exclusive breastfeeding on neurodevelopment. The findings from various studies indicate the strong effect of breastfeeding on the neurodevelopmental outcomes as compared to the administration of other milk formulas. Another finding from previous literature, the favorable events that are obtained from breastfeeding were also found to be sustained to the school-age of these children. On the other hand, evidence regarding the efficacy of breastfeeding on brain development in preterm infants is still poor. As a result, further studies are needed to furtherly validate this point. The main mechanisms by which favorable neurodevelopmental outcomes are obtained following breastfeeding are the nutritional values that are found within the breast milk along with the physical interactions between the mothers and infants during breastfeeding.
\end{abstract}

Keywords: Breastfeeding, Neurodevelopment, Pediatrics, Effectiveness

\section{INTRODUCTION}

Evidence in the literature indicates that individuals who have higher intelligence scores than others significantly have better socioeconomic statuses, enhanced educational outcomes, and higher success rates in their careers. ${ }^{1-4}$ Back in 1929, an investigation by Hoefer et al was the first to demonstrate that children that were breastfed had significantly higher cognitive abilities than other children who were not. ${ }^{5}$ Many advantages have been reported among the various studies in the literature about the efficacy of breastfeeding on brain development, which is mainly attributable to the abundant presence of bioactive compounds, and the essential fatty acids in the breast milk, moreover, it has been proven that breastfeeding enhance the long-term outcomes in these children. ${ }^{6-9}$ In this context, arachidonic acid and docosahexaenoic acid are two fatty acids that were previously reported to be associated with the developmental outcomes of the retina, nerve cells and brain. Both of these amino acids have also been found in breast milk and are not present in other milk formulas as cow's milk that are usually administered to newborns. In this context, evidence from previous studies in the literature has indicated that motor functions 
and eyesight abilities were significantly improved among children that were breastfed during the early months of their lives. ${ }^{10-16}$ It should be noted that not many studies have investigated the efficacy of exclusive breastfeeding in enhancing neurological outcomes, and many studies do not differentiate between the different milk formulas. Therefore, in the present literature review, we aim to discuss the impact of exclusive breastfeeding on neurodevelopment according to studies in the literature.

We performed an extensive literature search of the Medline, Cochrane, and EMBASE databases on $18^{\text {th }}$ July 2021 using the medical subject headings (MeSH) or a combination of all possible related terms. This was followed by the manual search for papers in Google Scholar and the reference lists of the initially included papers. Papers discussing exclusive breastfeeding and its impact on neurodevelopment were screened for relevant information. We did not pose any limits on date, language, age of participants, or publication type.

\section{DISCUSSION}

\section{Impact of breastfeeding on neurodevelopment}

Many studies in the literature have been previously published research related to the impact and outcomes of exclusive breastfeeding on neurodevelopment and cognitive abilities. Most of the studies in the literature are observational. However, other randomized trials could also be identified in the current literature because they have stronger evidence related to the validation of the effectiveness of the used interventions. In general, there is strong evidence supporting the enhanced outcomes of breastfeeding on the cognitive abilities of the breastfed children. Nevertheless, evidence regarding other neurological-related outcomes as emotional, behavioral, and social functions is still poor. In a previous cohort investigation by Jedrychowski et al, the authors aimed to assess the effectiveness of exclusive breastfeeding on the neurodevelopmental outcomes in a long study that lasted for 7 years of follow-up. ${ }^{17}$ The authors reported that in children with exclusive breastfeeding, the intelligence quotients (IQs) were higher by 2.1 points than other children that were administered mixed breastfeeding formulas. Besides, they reported that the acquired enhancements in the IQ in the exclusive breastfeeding group were significantly maintained for the preschool age, although it was originally acquired within the first year of life. These results are in line with the previous recommendations by the world health organization that all children should administer exclusive breastfeeding during the first six months of life. ${ }^{18-20}$ Another investigation by Jing et al also has shown that children that were breastfed by their mothers were significantly observed to have enhanced brain electrical activities during infancy when compared to other children that were administered soy or cow's milk. ${ }^{21}$ In this context, the authors attribute such events to the abundant presence of the omega-3 polyunsaturated fatty acids and other biological compounds that are naturally found in breast milk than other formulas observed in their cohorts. In addition, other studies have demonstrated that breastfeeding provides the breastfed children with significant maternal attachments which might also be the main cause behind the enhanced cognitive abilities in these children. This has been also associated with better psychological outcomes on children later on in their life. $^{22-25}$ It is logical because, during breastfeeding, there are increased amounts of physical stimulation, mutual touch, and mother's gaze than what can be observed during bottle feeding. Also, maternal intelligence might be significantly associated with breastfeeding, although it was not adequately measured among studies in the literature.

A previous 10-year Australian study has assessed the language abilities of their included children at the age of 10 years old, and its association with the duration of breastfeeding during childhood. The authors of this study have reported that increased scores of the used language ability test were higher than average by two points for children that were exclusively fed by breast milk for at least six months, and higher than other children that were administered other milk formulas. ${ }^{26}$ This is also consistent with a previous meta-analysis that combined the results of 20 controlled investigations, and they showed that after adjustment of the cofactors, children that were treated with breast milk formulas had significantly higher cognitive-developmental scores by 2.3 points than other children that were treated with formula-feeding modalities. $^{27}$ No significant heterogeneity was also reported by the meta-analysis for the included investigations. As previously mentioned, the observed outcomes were noticed during the early months following the initiation of breastfeeding, and the favorable outcomes were furtherly maintained during the following years, as well. On the other hand, a previous large cohort investigation by Der et al has reported that breastfeeding had no or little impact on the intellectual and cognitive abilities of the breastfed children. ${ }^{28}$ However, the authors of the study considered the effect of breastfeeding only, and compared their cohorts being on whether or not they were breastfed, and no correlation with exclusive breastfeeding was established in this study. Consequently, the authors were vague about the definition of breastfeeding as some of the "breastfeeding groups" might have occasionally administered mixed formulas with very little breast milk during the study period. Accordingly, the results of this investigation should be carefully interpreted, and we suggest that other aforementioned investigations have stronger evidence.

It should be noted that the evidence regarding the association between gestational age and cognitive abilities of children is still poor in the current literature. Among the different studies in the literature, gestational age is only considered in cases of assessing delivery complications, rates of premature infants, or in the determination of obesity among infants. Accordingly, the 
evidence regarding the association between gestational age and cognitive abilities in breastfed infants is still not clear within the current literature. As it was previously demonstrated that the gestational age of newborns is significantly associated with overweight. It could be suggested that increased gestational age might negatively impact the cognitive and psychological abilities of the corresponding children.

\section{Brain development and proposed mechanisms}

In the previous section, we have discussed the potential developmental outcomes that breastfeeding might have on the brains of the corresponding children. Although these results are well-proven among the different studies in the literature. Evidence regarding the efficacy of the modality on many infants is still poor within the current literature. Studies show that infants who have increased nutritional requirements were born at an early stage from brain development, were preterm infants that were born at $<32$ weeks of gestation or have increased risk of developing neurological impairments are more prone to not benefit from breastfeeding regarding the brain development. $^{29-31}$ As a result of the potential complications that might occur to these children at birth, they are usually admitted to the hospital to be given full care. Therefore, they are not usually breast or bottle-fed, but they are fed with a feeding tube (Figure 1). Accordingly, studies that have focused on the outcomes of breastfeeding in these children should have extended their outcomes beyond hospital discharge and investigate the effectiveness of breastfeeding on the neurodevelopmental outcomes of children after they were discharged from the intensive care units for further validation. Although some studies have reported that breastfeeding might have beneficial effects on preterm infants, these studies were limited by the small samples and the design limitations. ${ }^{32}$ Another important factor that should be considered in such cases is the age when the assessment was conducted. This is logical because assessment of the neurodevelopmental outcomes in late infancy might be too early to detect any favorable events regarding cognitive abilities. On the other hand, a limited number of studies have assessed the outcomes in large cohorts of preschool preterm children. For instance, an Australian investigation recruited 180 preterm infants (born at $<30$ weeks of gestation), and assessed the effect of initiating breastfeeding to these children during the first month from neonatal intensive care units admission on the cognitive functions at 2 and 7 years old. The authors of the study reported that breastmilk intake was not associated with favorable cognitive and motor functions at 2 years old. However, it was significantly proportionally associated with increased IQ and motor functions at 7 years old in their included population. ${ }^{33}$ Another randomized controlled trial was also published to elaborate more on the effectiveness of breast milk initiation during hospitalization of preterm infants. The donor milk for improved neurodevelopmental outcomes (DoMINO) trial has recruited 840 infants (that were born with $<1500 \mathrm{~g}$ ) and randomized them into receiving either pasteurized donor milk or preterm formula as supplementation to their mother's milk whenever needed. The authors reported that the rate of infants that had cognitive impairments (based on Bayley scale scores) was higher in the population receiving donor's milk than the other group. On the other hand, the authors reported that no significant differences were noticed between the two groups in terms of language, cognitive and motor scores at 18 months from initiating the feeding modality. It is worth noting that the authors also have shown that the rate of necrotizing enterocolitis was lower in the infants that received donor milk supplements, which might have increased the survival rates in these infants. ${ }^{34}$ Accordingly, it has been concluded that donor milk is not as beneficial as mother's milk in terms of brain development and the relevant outcomes.

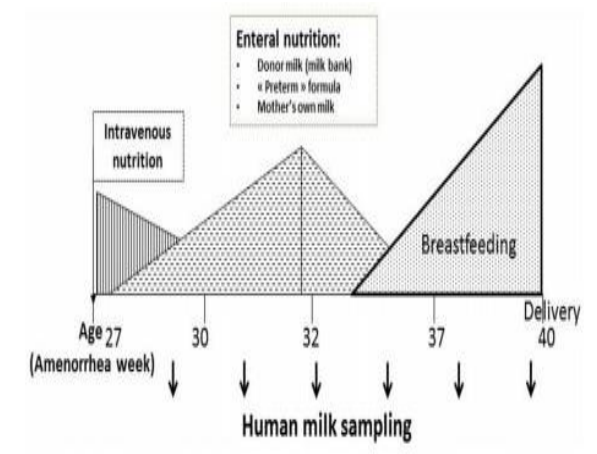

Figure 1: Feeding of preterm infants during the first
months of life. ${ }^{39}$

It is widely known that breastfeeding is a common practice among different mammals that allows for adequate transfer of the nutritional and immunological components to the infants from their mothers. ${ }^{35}$ Besides, it has been demonstrated that, as the infantile brain begins to develop within the first months of life, the nutritional modality that is administered to these infants hugely impacts the development of their brains. Accordingly, the enhanced neurodevelopmental outcomes that could be observed with the breastfed infants are most probably attributable to the factors that are or are not related to the nutritional components that are present in the human milk and not present in other formulas. However, evidence regarding the adequate identification of the nutritional components of breast milk is still poor among the different studies in the literature. ${ }^{36,37}$ Another important factor would be the extended maternal care offered by breastfeeding, which reinforces the interactions between the mother and the baby and with the surrounding environment leading to enhanced neurodevelopmental outcomes and cognitive capabilities. Therefore, it has been demonstrated that the effectiveness of breastfeeding extends beyond the nutritional components that are present in breast milk than other milk formulas, indicating the importance of mother-to-infant interactions during the very few months of life. ${ }^{38}$ 


\section{CONCLUSION}

The findings from various studies indicate the strong effect of breastfeeding on the neurodevelopmental outcomes as compared to the administration of other milk formulas. The favorable events that are obtained from breastfeeding were also found to be sustained to the school-age of these children. With evidence regarding the efficacy of breastfeeding on brain development in preterm infants is still poor, further studies are needed to furtherly validate this point. The main mechanisms by which favorable neurodevelopmental outcomes are obtained following breastfeeding are the nutritional values that are found within the breast milk along with the physical interactions between the mothers and infants during breastfeeding.

\section{Funding: No funding sources \\ Conflict of interest: None declared \\ Ethical approval: Not required}

\section{REFERENCES}

1. Deary IJ. Cognitive epidemiology: Its rise, its current issues, and its challenges. Personality and individual differences. 2010;49(4):337-43.

2. Hunter JE. Cognitive ability, cognitive aptitudes, job knowledge, and job performance. $\mathrm{J}$ vocational behavior. 1986;29(3):340-62.

3. McCall RB. Childhood IQ's as predictors of adult educational and occupational status. Science. 1977;197(4302):482-3.

4. Moffitt TE, Gabrielli WF, Mednick SA, Schulsinger F. Socioeconomic status, IQ, and delinquency. J Abnormal Psychology. 1981;90(2):152.

5. Hoefer C, Hardy MC. Later development of breast fed and artifically fed infants: comparison of physical and mental growth. J Am Med Assoc. 1929;92(8):615-9.

6. Crawford MA. The role of essential fatty acids in neural development: implications for perinatal nutrition. Am j clin nutr. 1993;57(5):703S-10S.

7. Makrides M, Neumann MA, Byard RW, Simmer K, Gibson RA. Fatty acid composition of brain, retina, and erythrocytes in breast-and formula-fed infants. Am j clin nutrition. 1994;60(2):189-94.

8. Neuringer M, Connor WE. n-3 fatty acids in the brain and retina: evidence for their essentiality. Nutrition reviews. 1986;44(9):285-94.

9. Ghozy S, Tran L, Naveed S. Association of breastfeeding status with risk of autism spectrum disorder: A systematic review, dose-response analysis and meta-analysis. Asian $\mathrm{J}$ Psychiatr. 2020;48:101916.

10. Agostoni C, Marangoni F, Lammardo A, Giovannini M, Riva E, Galli C. Breastfeeding duration, milk fat composition and developmental indices at 1 year of life among breastfed infants. Prostaglandins, Leukotrienes and Essential Fatty Acids (PLEFA). 2001;64(2):105-9.
11. Birch E, Birch D, Hoffman D, Uauy R. Dietary essential fatty acid supply and visual acuity development. Investigative ophthalmol visual sci. 1992;33(11):3242-53.

12. Carlson SE, Werkman S, Rhodes PG, Tolley E. Visual-acuity development in healthy preterm infants: effect of marine-oil supplementation. Am j clin nutrition. 1993;58(1):35-42.

13. Farquharson J, Jamieson EC, Abbasi KA, Patrick W, Logan RW, Cockburn F. Effect of diet on the fatty acid composition of the major phospholipids of infant cerebral cortex. Arch disease childhood. 1995;72(3):198-203.

14. Lundqvist-Persson C, Lau G, Nordin P, Strandvik B, Sabel KG. Early behaviour and development in breast-fed premature infants are influenced by omega-6 and omega-3 fatty acid status. Early Hum Dev. 2010;86(7):407-12.

15. Uauy RD, Birch DG, Birch EE, Tyson JE, Hoffman DR. Effect of dietary omega-3 fatty acids on retinal function of very-low-birth-weight neonates. Pediatric Res. 1990;28(5):485-92.

16. Son PT, Reda A, Viet DC. Exchange transfusion in the management of critical pertussis in young infants: a case series. Vox Sanguinis. 2021.

17. Jedrychowski W, Perera F, Jankowski J. Effect of exclusive breastfeeding on the development of children's cognitive function in the Krakow prospective birth cohort study. Eur J Pediatr. 2012;171(1):151-8.

18. Growth WWGoI. An evaluation of infant growth. In: World Health Organization Geneva. 1994.

19. Kramer MS, Kakuma R. The optimal duration of exclusive breastfeeding: a systematic review. Adv in experimental med biol. 2004;554:63-77.

20. Pham TS, Reda A, Ngan Nguyen TT. Blood exchange transfusion in viral hepatitis in a small infant: a case report. Transfusion Apheresis Sci. 2020;59(6): 102907.

21. Jing H, Gilchrist JM, Badger TM, Pivik R. A longitudinal study of differences in electroencephalographic activity among breastfed, milk formula-fed, and soy formula-fed infants during the first year of life. Early human development. 2010;86(2):119-25.

22. Jansen J, de Weerth C, Riksen-Walraven JM. Breastfeeding and the mother-infant relationship-a review. Developmental review. 2008;28(4):503-21.

23. Meedya S, Fahy K, Kable A. Factors that positively influence breastfeeding duration to 6 months: a literature review. Women and birth. 2010;23(4):13545 .

24. Morley R, Cole T, Powell R, Lucas A. Mother's choice to provide breast milk and developmental outcome. Arch disease childhood. 1988;63(11):13825.

25. Thieu H, Bach Dat B, Nam NH. Antibiotic resistance of Helicobacter pylori infection in a children's hospital in Vietnam: prevalence and associated factors. Minerva medica. 2020;111(5):498-501. 
26. Whitehouse AJ, Robinson M, Li J, Oddy WH. Duration of breast feeding and language ability in middle childhood. Paediatr perinatal epidemiol. 2011;25(1):44-52.

27. Anderson JW, Johnstone BM, Remley DT. Breastfeeding and cognitive development: a meta-analysis. Am j clin nutr. 1999;70(4):525-35.

28. Der G, Batty G, Deary I. Effect of breast feeding on intelligence in children: Prospective study, sibling pairs analysis, and meta-analysis. BMJ (Clinical research ed). 2006;333:945.

29. Smyser CD, Kidokoro H, Inder TE. Magnetic resonance imaging of the brain at term equivalent age in extremely premature neonates: to scan or not to scan? J paediatr child health. 2012;48(9):794-800.

30. Krebs NF. American Academy of Pediatrics Committee on Nutrition. Prevention of pediatric overweight and obesity. Pediatrics. 2003;112:424-30.

31. Aarnoudse-Moens CS, Weisglas-Kuperus N, van Goudoever JB, Oosterlaan J. Meta-analysis of neurobehavioral outcomes in very preterm and/or very low birth weight children. Pediatrics. 2009;124(2):717-8.

32. Koo W, Tank S, Martin S, Shi R. Human milk and neurodevelopment in children with very low birth weight: a systematic review. Nutrition journal. 2014;13:94.

33. Belfort MB, Anderson PJ, Nowak VA. Breast Milk Feeding, Brain Development, and Neurocognitive Outcomes: A 7-Year Longitudinal Study in Infants Born at Less Than 30 Weeks' Gestation. J pediatr. 2016;177:133-9.e131.
34. O'Connor DL, Gibbins S, Kiss A. Effect of Supplemental Donor Human Milk Compared With Preterm Formula on Neurodevelopment of Very Low-Birth-Weight Infants at 18 Months: A Randomized Clinical Trial. Jama. 2016;316(18):1897-905.

35. Power ML, Schulkin J. Milk: the biology of lactation. JHU Press. 2016.

36. Collins CT, Gibson RA, Anderson PJ. Neurodevelopmental outcomes at 7 years' corrected age in preterm infants who were fed high-dose docosahexaenoic acid to term equivalent: a follow-up of a randomised controlled trial. BMJ open. 2015;5(3):e007314.

37. Jasani B, Simmer K, Patole SK, Rao SC. Long chain polyunsaturated fatty acid supplementation in infants born at term. The Cochrane database of systematic reviews. 2017;3(3):Cd000376.

38. Smith JP, Ellwood M. Feeding patterns and emotional care in breastfed infants. Social Indicators Res. 2011;101(2):227-31.

39. Boquien C-Y. Human Milk: An Ideal Food for Nutrition of Preterm Newborn. Frontiers in Pediatrics. 2018;6:295.

Cite this article as: Alghamdi SS, Alruwaili MS, Alanazi NH, Alalmaei AZ, Alshammari RS, Tuwalah FFB et al. Exclusive breastfeeding and its impact on neurodevelopment. Int J Community Med Public Health 2021;8:4128-32. 